\title{
Increased c-kit (CD117) expression in malignant mammary phyllodes tumors
}

\author{
Gary MK Tse ${ }^{1}$, Thomas C Putti ${ }^{2}$, Philip CW Lui ${ }^{3}$, Anthony WI Lo ${ }^{1}$, Richard A Scolyer ${ }^{4,5}$, \\ Bonita KB Law ${ }^{6}$, Rooshdiya Karim ${ }^{4,5}$ and C Soon Lee ${ }^{4,5}$ \\ ${ }^{1}$ Department of Anatomical and Cellular Pathology, Prince of Wales Hospital, Chinese University of Hong \\ Kong, Hong Kong; ${ }^{2}$ Department of Pathology, National University Hospital, Singapore; ${ }^{3}$ Department of \\ Pathology, United Christian Hospital, Hong Kong; ${ }^{4}$ Department of Pathology, University of Sydney, Sydney, \\ Australia; ${ }^{5}$ Department of Anatomical Pathology, Royal Prince Alfred Hospital, Sydney, Australia and \\ ${ }^{6}$ Department of Surgery, Prince of Wales Hospital, Chinese University of Hong Kong, Hong Kong
}

\begin{abstract}
Mammary phyllodes tumors are uncommon stromal neoplasms, and are divided into benign, borderline and malignant groups basing on histologic criteria. While benign phyllodes tumors may recur, borderline phyllodes tumors show higher propensity to recur locally and rarely metastasize, and malignant phyllodes tumors show even higher chances of local recurrences or distant metastases. c-kit is a proto-oncogene that encodes a tyrosine kinase receptor (CD117) and is a marker for gastrointestinal stromal tumors (GIST). With the advent of therapeutic agent targeted at this receptor for GIST, we investigated 179 phyllodes tumors (101 benign, 50 borderline, 28 malignant) for c-kit expression using immunohistochemistry. The staining was compared to the degree of malignancy, and to the degree of stromal cellularity, mitotic activity, nuclear pleomorphism and stromal overgrowth. The overall positive rate for c-kit was $29 \%(52 / 179)$ and $17 \%(17 / 101), 24 \%(12 / 50)$ and $46 \%$ (13/28), respectively, for benign, borderline malignant and frank malignant phyllodes and the differences between all categories were significant $\left(\chi^{2}=13.844, P=0.001\right)$. In mammary phyllodes tumors, there was increasing c-kit expression with increasing degree of malignancy, up to $46 \%$ in malignant cases. This provides strong evidence that c-kit receptor mediated tyrosine kinase involvement in the pathogenesis of phyllodes tumors, and the therapeutic agent, STI571, Glivec, may be a potentially useful drug for its management. Modern Pathology (2004) 17, 827-831, advance online publication, 26 March 2004; doi:10.1038/modpathol.3800125
\end{abstract}

Keywords: breast; phyllodes tumor; c-kit; CD117; immunohistochemistry

Mammary phyllodes tumor is an uncommon stromal epithelial neoplasm, constituting $0.3-0.5 \%$ of all breast lesions. ${ }^{1-5}$ It usually occurs in middle-aged females, but can rarely occur in a younger age group and in men. Based on a combination of histologic criteria, which includes stromal cellularity, nuclear pleomorphism, mitotic rate, stromal overgrowth and the margin status (whether infiltrative or rounded), phyllodes tumors can be divided into benign, borderline and malignant groups. ${ }^{1,6}$ While all groups of phyllodes tumors show a propensity to recur locally, the borderline and malignant groups may also metastasize to other visceral organs.

c-kit is a proto-oncogene that encodes a tyrosine kinase receptor (CD117) and is a marker for bone

Correspondence: GMK Tse, FRCPC, Department of Anatomical and Cellular Pathology, Prince of Wales Hospital, Ngan Shing Street, Shatin, NT, HKSAR, China.

E-mail: garytse@cuhk.edu.hk

Received 2 November 2003; revised and accepted 18 December 2003; published online 26 March 2004 marrow progenitor cells ${ }^{7}$ and gastrointestinal stromal tumors. ${ }^{8}$ The expression of c-kit in mammary phyllodes tumor has not been widely investigated, ${ }^{9,10}$ with the reported case number being less than 50. Another stromal tumor of the breast, termed 'periductal stromal tumor', has also been demonstrated to express c-kit. ${ }^{11}$ Given the advent of specific therapy targeted at this tyrosine kinase receptor, and the known propensity of mammary phyllodes tumors to recur or metastasize, and with preliminary results suggesting increased c-kit expression in malignant phyllodes tumor, ${ }^{9}$ it is imperative to follow on this observation using a larger series. This paper presents the findings of an international multicenter study of c-kit expression in 179 phyllodes tumors, the largest series documented in the literature.

\section{Materials and methods}

The histologic files of the four participating institutions were searched for phyllodes tumors of the 
breast. The number of years searched ranged from 4 to 15 years. The paraffin blocks were retrieved and $4-\mu \mathrm{m}$ slides were prepared routinely and stained with hematoxylin and eosin (H\&E). All the slides were reviewed for the following histologic parameters: (1) stromal cellularity; (2) nuclear pleomorphism; (3) stromal overgrowth; (4) mitotic rate; and (5) margin of the tumor (whether infiltrative or rounded). Parameters (1) and (2) were graded as low/mild, moderate or severe; stromal overgrowth was graded as present or absent; and the mitotic count was expressed as the number of mitotic figures per 10 high-power fields $(\times 400$, Nikon Labophot, field area $0.19 \mathrm{~mm}^{2}$ ). As previously described, ${ }^{12}$ a diagnosis of benign phyllodes tumor was made when there was low cellularity, no stromal overgrowth, mild pleomorphism, a rounded margin and a mitotic count of 2 or less per 10 highpower fields. Malignant phyllodes tumor was diagnosed when the mitotic count was 5 or more per 10 high-power fields together with stromal overgrowth and an infiltrative margin. Phyllodes tumor of borderline malignancy was diagnosed when the criteria for malignant phyllodes tumor were not totally fulfilled.

For the assessment of c-kit expression, a representative slide from each case was stained using an antibody against c-kit (Novacastra, UK; $1: 40$ ) with the avidin-biotin method. The staining of the stromal cells was assessed, using the cytoplasmic staining of the breast epithelium as internal control. The staining was assessed for intensity, graded as 0 if there was no staining, low, intermediate or high if the staining was weaker than, the same as or stronger than that of the epithelium. The percentage of the cells showed that staining was also assessed. The phyllodes tumor was considered positive for c-kit if there was moderate-to-strong cytoplasmic staining in $20 \%$ or more of the stromal cells.

For the statistical analysis, ANOVA with post hoc comparisons was used to determine differences in patient ages and tumor sizes between benign, borderline malignant and frankly malignant phyllodes tumors. $\chi^{2}$ test and $\chi^{2}$ test for trend were used to determine the difference between the expression of c-kit among the three categories of phyllodes tumors. The statistically significant level was set at $5 \%$.

\section{Results}

A total of 179 cases of phyllodes tumors were included in this series. These tumors were obtained from 173 patients, all of whom were women. Among all the 179 tumors, there were 157 phyllodes tumors without known recurrence, seven initial tumors that later recurred, 13 first recurrent tumors and two second recurrences. In total, there were 101 benign phyllodes tumors; 50 phyllodes tumors were of borderline malignancy and 28 frankly malignant phyllodes tumors. The overall patient age range was 14-77 years (mean 42 years), and the tumor size range was $0.8-22 \mathrm{~cm}$ (mean $4.2 \mathrm{~cm}$ ). In all, 89 phyllodes tumors were from the left breast, 88 were from the right, and in two cases, the tumor sites were unknown. For the ethnic group distribution, 105 patients were Chinese, 30 were Caucasians, 12 were Malaysians, seven were Indians, seven were Indochinese, and 12 patients whose ethnic groups were not known.

For the benign phyllodes tumors, the patient age range was 14-60 years (mean 39.7 years). The tumor size range was $0.8-22 \mathrm{~cm}$ (mean $4 \mathrm{~cm}$ ). For the phyllodes tumors of borderline malignancy, the patient age range was 15-77 years (mean 45.4 years), and the tumor size range was $1-20 \mathrm{~cm}$ (mean $5.5 \mathrm{~cm}$ ). For the malignant phyllodes tumors, the patient age range was 19-70 years (mean 45.9 years), and the tumor size range was $1.5-22 \mathrm{~cm}$ (mean $6.7 \mathrm{~cm}$ ). There was an increase in the average patient age from benign to borderline malignant to frankly malignant phyllodes tumors and the differences were statistically significant $(F=6.782, P=0.001)$. The tumor sizes also showed a similar trend, with increasing degree of malignancy associated with increasing sizes. The difference between the benign and borderline malignant groups was statistically significant $(P=0.030)$, but not between the latter group and the malignant group $(P=0.996)$.

For the expression of c-kit by immunohistochemistry, the overall positive rate was 52 out of 179 $(29 \%)$ phyllodes tumors. For the different categories of phyllodes tumors, the respective positive rates for benign, borderline malignancy and frankly malignant phyllodes tumors were 17 of 101 (16.8\%), 12 of $50(24 \%)$ and 13 of $28(46.4 \%)$ (Table 1, Figures 1 and 2). Using $\chi^{2}$ test, the differences between all categories were significant $\left(\chi^{2}=13.844, P=0.001\right)$. Hence, there is a statistically significant increase in c-kit expression from benign to borderline to frankly malignant phyllodes tumors (Kendall's tau, $T=3.184, P=0.001$ ).

In our series, there were seven phyllodes tumors that later recurred, 13 first recurrences, two second recurrences and two with distant metastases. The expression of c-kit was noted in one of seven phyllodes tumors that eventually recurred, four of 13 first recurrences, none of the second recurrences and in one of two with distant metastases. There

Table 1 Expression of c-kit in phyllodes tumors of differing degrees of malignancy

\begin{tabular}{lcc}
\hline Type of PT & No. of cases (\%) & c-kit positive (\%) \\
\hline All PT (\%) & $179(100 \%)$ & $52(29 \%)$ \\
Benign PT (\%) & 101 & $17(17 \%)$ \\
Borderline PT (\%) & 50 & $12(24 \%)$ \\
Malignant PT (\%) & 28 & $13(46 \%)$ \\
\hline
\end{tabular}

$\mathrm{PT}=$ phyllodes tumors; $\%=$ percentage. 


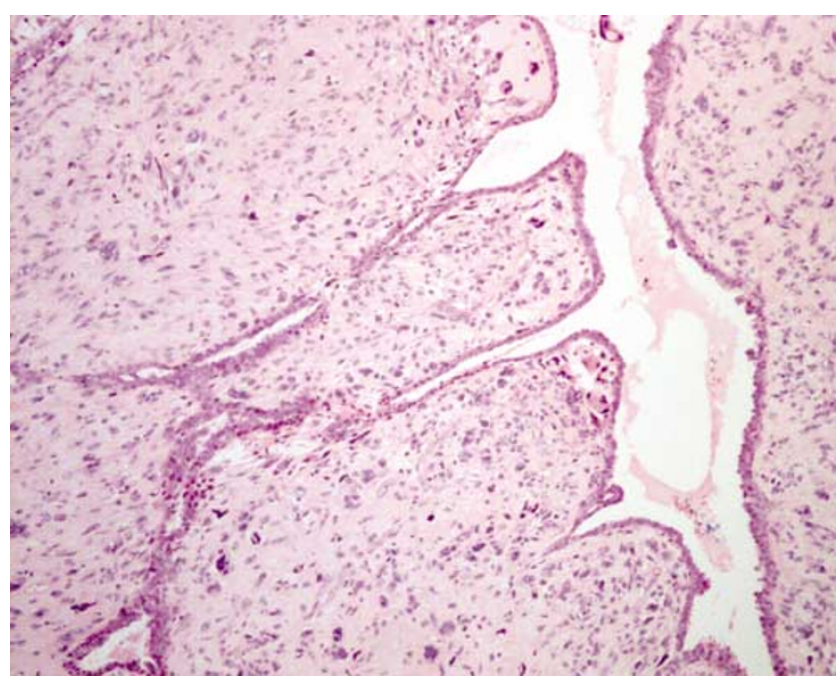

Figure 1 Photomicrograph showing a malignant phyllodes tumor with high stroma cellularity and some multinucleated stromal giant cells $(\mathrm{H} \& \mathrm{E}, \times 100)$.

was no relationship between c-kit expression and recurrence.

In addition, several unique morphological features were observed. Although the general trend of increasing staining was observed with increasing degree of malignancy, staining was also present in some benign phyllodes tumors. In all categories of phyllodes tumors, the distribution of c-kit expressing stromal cells was located mostly in the subepithelial areas where there were condensations of the stromal cells with a focal increase in cellularity. In those cases in which the occasional stromal giant cells were observed, irrespective of the degree of the malignancy of the phyllodes tumors, these giant cells tended to show positive staining.

\section{Discussion}

The c-kit gene encodes a transmembrane tyrosine kinase receptor protein. In the breast, it has been found to be expressed in the normal breast ductal epithelium and myoepithelial cells, with reduced expression in benign breast lesions and carcinoma. ${ }^{13-16}$ Previous studies that focused on carcinoma of the breast showed markedly reduced expression of c-kit in carcinoma, ${ }^{13-16}$ borderline epithelial lesions ${ }^{16}$ and in the breast cancer cell line MCF 7 , in which c-kit was thought to mediate inhibitory signals for the growth of cancer cells in the breast. ${ }^{17}$ In one study, however, it was found that poor prognosis cancers of the breast showed positivity for c-kit by immunostaining in the majority of the cases $(82 \%),{ }^{18}$ illustrating that this issue is not yet settled.

The expression of c-kit in phyllodes tumor is even less well studied. In the most recent large study on

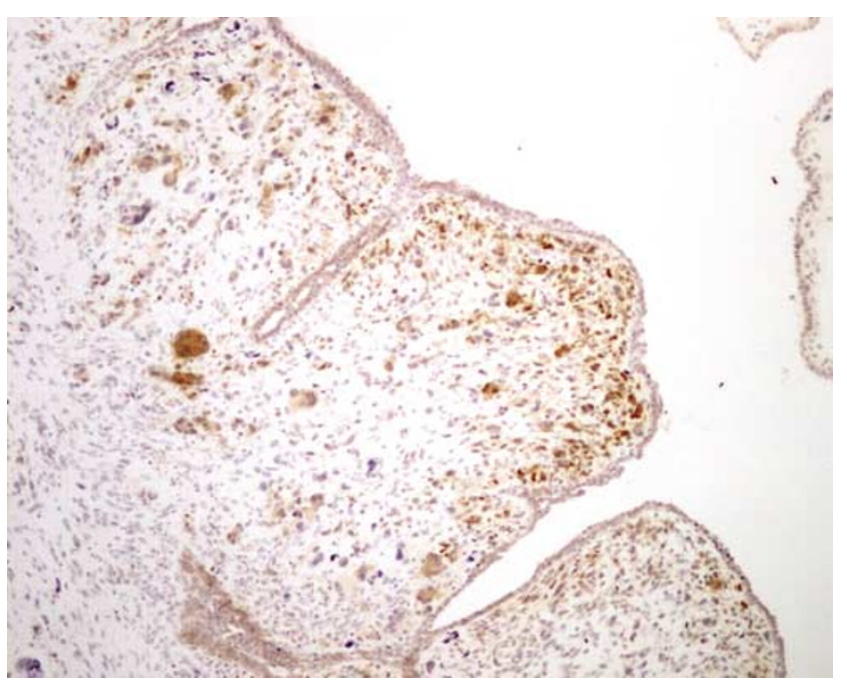

Figure 2 Photomicrograph showing the same malignant phyllodes tumor with positive staining of subepithelial stromal cells and in the multinucleated stromal giant cells $(\times 100)$.

phyllodes tumors, the c-kit expression rate was cited to be $50 \%$ for malignant phyllodes tumors and $5 \%$ for benign phyllodes tumors by immunohistochemistry. ${ }^{9}$ In contrast, another study ${ }^{16}$ failed to demonstrate any c-kit expression in the borderline malignant and frankly malignant phyllodes tumors. These apparently conflicting results in the literature require further clarification with a larger series as the therapeutic agent, STI571 (Glivec, Novartis), directed towards c-kit expressing tyrosine kinase is useful against gastrointestinal stromal tumors. STI571 appeared to be specific for GIST with KIT mutation, as in the EORTC trial, ${ }^{19}$ it was not effective to all non-GIST soft-tissue tumors that did not express c-kit. Furthermore, a recent study demonstrated that in late-stage metastatic GIST, different mutations within the KIT gene resulted in different STI571 effectiveness. ${ }^{20}$ In malignant phyllodes tumors, as the lesional cells are also stromal in origin, an understanding or a more comprehensive assessment of the c-kit expression in phyllodes tumors may be crucial for possible future use of STI571 in these tumors. However, as the effectiveness in other soft-tissue tumor of STI571 has not been conclusively demonstrated, it is still too early to speculate on its role in mammary phyllodes tumors.

In the current study, which is to our knowledge the largest series documented in the literature, it is shown that the expression of c-kit in phyllodes tumors as indicated by strong immunostaining progressively increased from benign to malignant tumors. This finding is similar to that seen in two other recent studies, ${ }^{9,10}$ and confirms the previous observations of increased c-kit expression with increasing degrees of malignancy. In another study, c-kit expression was documented in a group of stromal lesions of the breast that lacks definite 
phyllodes-type architecture. These lesions were classified as periductal stromal hyperplasia when the stromal component was benign, and as periductal stromal sarcoma when the stromal component shows malignancy. ${ }^{11}$ In that series, c-kit expression was reported in the periductal stromal sarcoma, but not in the periductal stromal hyperplasia, supporting the hypothesis that increased c-kit expression is associated with increasing degree of malignancy in stromal lesions.

Morphologically, it was observed that the expression of c-kit was mostly localized in the subepithelial areas of stromal condensation, where the stromal cellularity tends to be highest, and where mitoses also tend to be more frequent. This indicates that c-kit expression is highest in the more active areas of the stromal proliferation, and is consistent with the role of c-kit in cellular proliferation.

For phyllodes tumors with recurrences or metastases, there does not appear to be any trend for c-kit expression in relation to recurrences or even metastases. However, the small number of such cases does not allow any meaningful conclusions to be drawn. As the overall percentage of c-kit staining phyllodes tumors is low (overall $29 \%$, ranging from $16.8 \%$ for benign tumors to $46 \%$ in malignant phyllodes tumors), it is probable that the activation of tyrosine kinase mediated by c-kit is not the only mechanism by which proliferation occurs in phyllodes tumors, although it may be an important one. The expression of c-kit in benign phyllodes tumors suggests that the c-kit-dependent tyrosine kinasedriven proliferative activity may be an early event in the neoplastic pathway. In the current series, the observation of c-kit expression in some benign and borderline phyllodes tumors is in contrast to that found by others ${ }^{9,10}$ in which c-kit expression was observed only in malignant tumors. However, the higher rate of c-kit expression in malignant phyllodes tumors in our study was comparable to these series. ${ }^{9,10}$

In view of the action of the newly developed phenylaminopyrimidine derivative STI571 (Glivec, Novartis), which inhibits tyrosine kinase receptors, and its usefulness in gastrointestinal stromal tumors ${ }^{21}$ the drug may theoretically also be useful in the management of malignant phyllodes tumor, particularly with tumor recurrences or distant metastases.

In this study, we demonstrated increasing c-kit expression in mammary phyllodes tumors, with increasing degree of malignancy. In malignant phyllodes tumors, the rate of expression is up to $46 \%$, similar to that previously reported in the literature. This provides additional strong evidence that c-kit receptor-mediated tyrosine kinase activity may be involved early on in the pathogenesis of phyllodes tumors, and the new therapeutic agent, STI571, Glivec, may be a useful drug therapy for this disease, particularly in the tumor recurrences and advanced-stage disease.

\section{References}

1 Azzopardi JG. Sarcoma of the breast. In: Bennington J (eds). Problems in Breast Pathology, Major Problems in Pathology, Vol. II. WB Saunders: Philadelphia, 1979, pp. 355-359.

2 Palmer ML, De Risi DC, Pelikan A, et al. Treatment options and recurrence potential for cystosarcoma phyllodes. Surg Gynecol Obstet 1990;170:193-196.

3 Kario K, Meada S, Mizuno Y, et al. Phyllodes tumor of the breast: a clinicopathologic study of 34 cases. J Surg Oncol 1990;45:46-51.

4 Rowell MD, Perry RR, Hsiu JG, et al. Phyllodes tumors. Am J Surg 1993;165:376-379.

5 Cole-Beuglet C, Soriano R, Kurtz AB, et al. Ultrasound, X-ray mammography and histopathology of cystosarcoma phyllodes. Radiology 1983;146:481-486.

6 Rosen PP. Breast Pathology, 2nd edn. LippincottRaven: Philadelphia, 2000, pp. 176-197.

7 Ashman LK, Cambareri AC, To LB, et al. Expression of the YB5.B8 antigen (c-kit proto-oncogene product) in normal human bone marrow. Blood 1991;78:30-37.

8 Lasota J, Jasinski M, Sarlomo-Rikala M, et al. Mutations in exon 11 of c-Kit occur preferentially in malignant versus benign gastrointestinal stromal tumors and do not occur in leiomyomas or leiomyosarcomas. Am J Pathol 1999;154:53-60.

9 Sawyer EJ, Poulsom R, Hunt FT, et al. Malignant phyllodes tumors show stromal overexpression of c-myc and c-kit. J Pathol 2003;200:59-64.

10 Chen CM, Chen CJ, Chang CL, et al. CD34, CD117, and actin expression in phyllodes tumor of the breast. J Surg Res 2000;94:84-91.

11 Burga AM, Tavassoli FA. Periductal stromal tumor: a rare lesion with low-grade sarcomatous behavior. Am J Surg Pathol 2003;27:343-348.

12 Tse GM, Putti TC, Kung FY, et al. Increased p53 protein expression in malignant mammary phyllodes tumors. Mod Pathol 2002;15:734-740.

13 Natali PG, Nicotra MR, Sures I, et al. Breast cancer is associated with loss of the c-kit oncogene product. Int J Cancer 1992;52:713-717.

14 Tsuura Y, Hiraki H, Watanabe K, et al. Preferential localization of c-kit product in tissue mast cells, basal cells of skin, epithelial cells of breast, small cell lung carcinoma and seminoma/dysgerminoma in human: immunohistochemical study on formalin-fixed, paraffin-embedded tissues. Virchows Arch 1994;424: 135-141.

15 Chui X, Egami H, Yamashita J, et al. Immunohistochemical expression of the c-kit proto-oncogene product in human malignant and non-malignant breast tissues. Br J Cancer 1996;3:1233-1236.

16 Tsuura Y, Suzuki T, Honma K, et al. Expression of c-kit protein in proliferative lesions of human breast: sexual difference and close association with phosphotyrosine status. J Cancer Res Clin Oncol 2002;128:239-246.

17 Nishida K, Tsukamoto T, Uchida K, et al. Introduction of the c-kit gene leads to growth suppression of a breast cancer cell line, MCF-7. Anticancer Res 1996;16: 3397-3402.

18 Palmu S, Soderstrom KO, Quazi K, et al. Expression of CKIT and HER-2 tyrosine kinase receptors in poorprognosis breast cancer. Anticancer Res 2002;22:411-414.

19 Verweij J, van Oosterom A, Blay JY, et al. Imatinib mesylate (STI-571 Glivec(R), Gleevec) is an active agent for gastrointestinal stromal tumours, but does 
not yield responses in other soft-tissue sarcomas that are unselected for a molecular target. Results from an EORTC Soft Tissue and Bone Sarcoma Group phase II study. Eur J Cancer 2003;39:2006-2011.

20 Heinrich MC, Corless CL, Demetri GD, et al. Kinase mutations and imatinib response in patients with metastatic gastrointestinal stromal tumor. J Clin Oncol 2003;21:4342-4349.

21 Heinrich MC, Blanke CD, Druker BJ, et al. Inhibition of KIT tyrosine kinase activity: a novel molecular approach to the treatment of KIT-positive malignancies. J Clin Oncol 2002;20:1692-1703. 